\title{
Protocolo de acabamento, texturização e polimento para restaurações diretas em resina composta
}

- Daniella Cristo Santin Departamento de Dentística, Endodontia e Materiais Odontológicos, Faculdade de Odontologia de Bauru, Universidade de São Paulo, Bauru, SP, Brasil • Cassiana Koch Scotti Departamento de Dentística, Endodontia e Materiais Odontológicos, Faculdade de Odontologia de Bauru, Universidade de São Paulo, Bauru, SP, Brasil • Marilia Mattar de Amoêdo Campos Velo Departamento de Dentística, Endodontia e Materiais Odontológicos, Faculdade de Odontologia de Bauru, Universidade de São Paulo, Bauru, SP, Brasil • Francielly da Silva Camim Departamento de Dentística, Endodontia e Materiais Odontológicos, Faculdade de Odontologia de Bauru, Universidade de São Paulo, Bauru, SP, Brasil • Rafael Francisco Lia Mondelli Departamento de Dentística, Endodontia e Materiais Odontológicos, Faculdade de Odontologia de Bauru, Universidade de São Paulo, Bauru, SP, Brasil • Juliana Fraga Soares Bombonatti Departamento de Dentística, Endodontia e Materiais Odontológicos, Faculdade de Odontologia de Bauru, Universidade de São Paulo, Bauru, SP, Brasil

RESUMO | O presente relato de caso clínico descreve uma abordagem conservadora para otimização de facetas diretas em resina composta. Para melhoria da anatomia e textura superficial das restaurações antigas, o tratamento baseou-se em um protocolo previsível e reprodutível de acabamento e polimento, o qual garantiu a obtenção de um sorriso equilibrado sem expor a paciente ao ciclo restaurador repetitivo. Inicialmente, a estratégia consistiu na remoção dos excessos de resina, definindo a anatomia primária dos dentes, seguindo a definição dos sulcos verticais e horizontais para mimetizar as características superficiais naturais, proporcionando superfície mais estética e favorável à reflexão de luz. Em adição, o polimento foi conduzido de modo a obter restaurações lisas e brilhantes, que proporcionam maior longevidade aos tratamentos restauradores estéticos.

DESCRITORES | Acabamento; Polimento; Resina composta; Estética.

ABSTRACT | Protocol of finishing, texturing and polishing for direct composite resin restorations - This clinical case report describes a conservative approach to optimize the composite resin direct veneer. In order to improve the anatomy and surface texture of previous restorations, the treatment was based on a predictable and reproducible finishing and polishing protocol which ensured a harmonic smile without exposing the patient to a repetitive restorative cycle. Firstly, the strategy consisted of removing excess resin, thus defining the primary anatomy of the teeth, following the definition of the vertical and horizontal grooves to mimic the natural surface characteristics, providing an aesthetic surface, favorable to light reflection. In addition, polishing was conducted in order to obtain smooth and shiny restorations, which provide greater longevity to the aesthetic restorative treatments.

DESCRIPTORS | Finishing; Polishing; Composite resin; Esthetic.

AUTOR CORRESPONDENTE | • Marilia Mattar de Amoêdo Campos Velo Faculdade de Odontologia de Bauru, Universidade de São Paulo • Alameda Dr. Octávio Pinheiro Brisolla, 9-75 Jardim Brasil Bauru, SP, Brazil • 17012-901 E-mail: mariliavelo@yahoo.com.br

- Received Dec 18, 2018 • Accepted Mar 4, 2019

- Dol http://dx.doi.org/10.11606/issn.2357-8041.clrd.2019.152964 


\section{INTRODUÇÃO}

Em função dos princípios de máxima conservação e mínima intervenção que norteiam o exercício da odontologia atual, as restaurações diretas em resina composta são consideradas estratégias de primeira eleição dentre as alternativas restauradoras para reestabelecimento ou otimização da estética em dentes anteriores. ${ }^{1,2}$ No entanto, a perda e/ou diminuição da percepção estética vem sendo relatada como principal razão para a substituição desse tipo de intervenção nos dentes anteriores. ${ }^{3,4}$

As etapas de acabamento e polimento das restaurações, muitas vezes negligenciadas pelos profissionais, são manobras clínicas essenciais e desafiadoras, capazes de otimizar a mimetização dos dentes naturais. ${ }^{5}$ A obtenção de uma superfície lisa e polida favorece tanto a saúde periodontal quanto a estética, além de melhorar a performance clínica das restaurações ao longo dos anos. ${ }^{6}$ Para minimizar a subjetividade do processo de obtenção de superfícies bem acabadas e polidas, o estabelecimento de um protocolo que proporcione resultados reprodutíveis é imprescindível, de forma a agregar todos os passos operatórios fundamentais na etapa de acabamento, texturização e polimento final.

$\mathrm{O}$ acabamento consiste na remoção dos excessos de resina composta, definindo a anatomia primária dos dentes. ${ }^{1,5}$ Já a texturização representa a determinação de caracterizações superficiais da estrutura dental. ${ }^{1}$ Por fim, o polimento é a suavização da rugosidade, resultando em uma superfície lisa e reflexiva, semelhante à dos dentes naturais. ${ }^{5,6}$

Diante do arsenal de materiais disponíveis no mercado para acabamento e polimento, a eleição de um sistema adequado é considerada um desafio. Assim, o objetivo deste relato de caso clínico é propor um protocolo previsível e reprodutível de acabamento, texturização e polimento de facetas diretas confeccionadas em resina composta.

\section{RELATO DE CASO}

Paciente, gênero feminino, 25 anos, insatisfeita com suas facetas em resina composta procurou atendimento odontológico. Durante o exame clínico foi observado sobrecontorno das restaurações dos dentes ântero-superiores que dificultavam a higienização devido a desadaptação marginal e perfil de emergência inadequado. A ausência de harmonia do sorriso com a face era evidenciada pela curva do sorriso invertida, ângulo reduzido entre as ameias incisais, além de dentes longos, planos e sem proporções ${ }^{7}$ (Figuras 1 e 2). Como as facetas estavam clinicamente adequadas em termos de volume, coloração e, principalmente, sem indícios de infiltração marginal, o tratamento proposto foi otimizar a anatomia e textura superficial das mesmas através de procedimentos de acabamento e polimento.

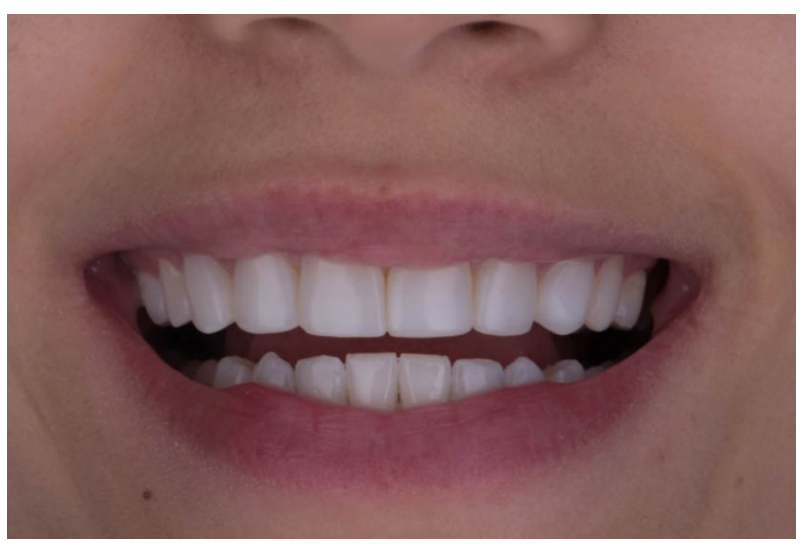

Figura 1 | Fotografia inicial do sorriso.

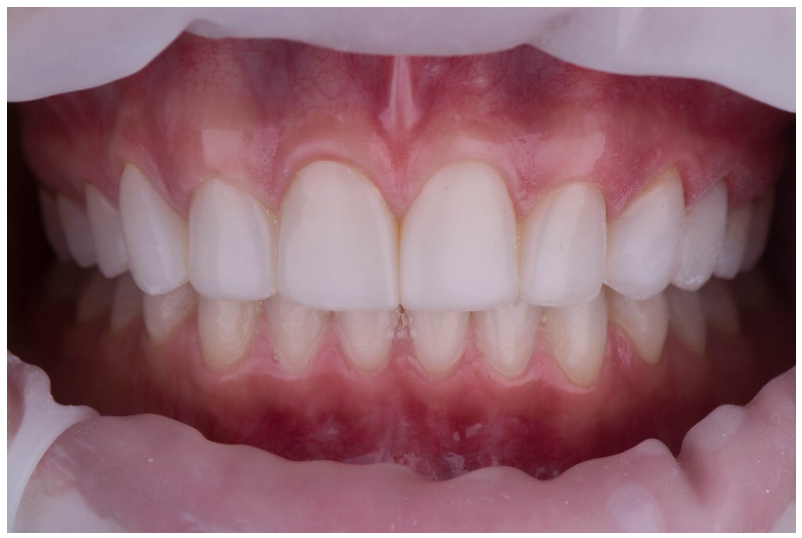

Figura 2 | Aspecto intraoral inicial do caso clínico. 


\section{Remoção de excessos cervicaís}

A intervenção foi iniciada removendo excessos cervicais de resina composta que acumulavam biofilme dental, induzindo a inflamação gengival. Para esta primeira etapa, uma lâmina cirúrgica \# 12D (Lamedid, Barueri, São Paulo, Brasil) montada em um cabo de bisturi foi posicionada no sulco gengival com inclinação de $45^{\circ}$ (Figura 3). Brocas multilaminadas de 12 e 30 lâminas (FG 7902, Jet Beavers, Ontário, Canadá) foram usadas para definir o correto perfil de emergência ${ }^{8}$ das restaurações (Figura 4).

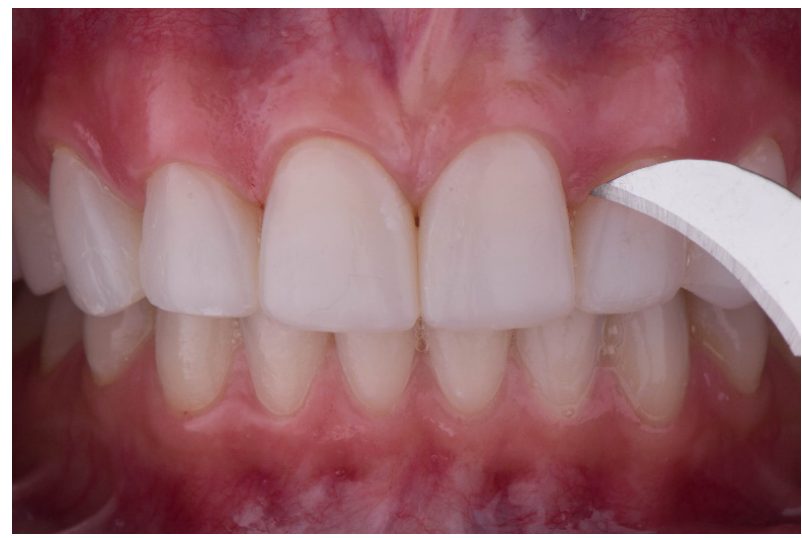

Figura 3 | Remoção dos excessos cervicais de resina composta.

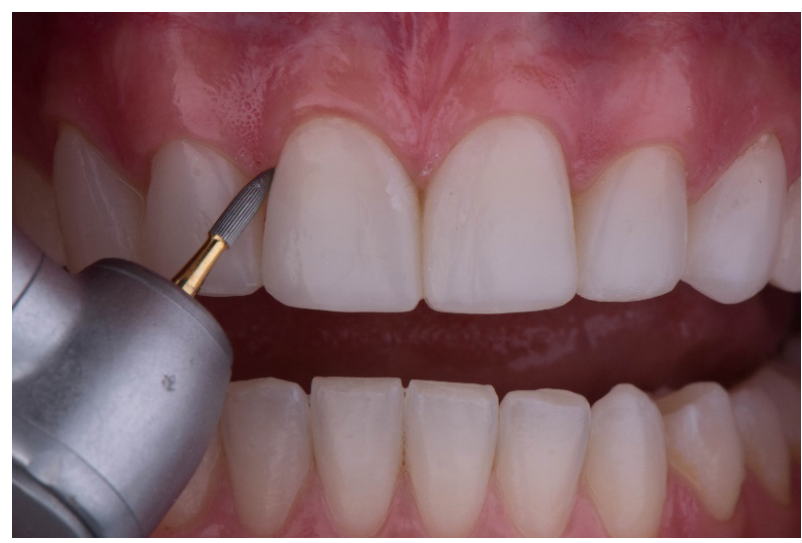

Figura 4 | Adequação do perfil de emergência das restaurações.

\section{Acabamento: anatomia primária}

Esta etapa baseia-se na redefinição da anatomia primária das facetas em relação ao tamanho e inclinação dos três terços da face vestibular (cervical, médio e incisal), posicionamento, espessura das bordas incisais e definição da bossa vestibular.

Sequencialmente, as ameias gengivais foram redefinidas com a utilização de tiras de lixa metálicas abrasivas de 2,5 mm de largura (Microdont, São Paulo, Brasil), evitando o rompimento do ponto de contato que deve estar localizado 3 a $5 \mathrm{~mm}$ de distância da crista óssea proximal para manutenção da saúde periodontal9. Em seguida, tiras de lixa abrasivas de granulações média e fina (KG Sorensen, São Paulo, Brasil) foram empregadas para refinar a lisura de superfície na região proximal.

Posteriormente, uma nova curva do sorriso ascendente para distal foi estabelecida com discos de lixa da granulação grossa (Sof-Lex laranja escuro, 3M ESPE, São Paulo, Brasil), apoiados sob a superfície incisal dos incisivos centrais superiores (Figura 5). Nesta etapa, especial atenção deve ser dada para que o abrasivo fique paralelo à borda incisal e centralizado com a linha média dentária, garantindo comprimento dental equivalente.

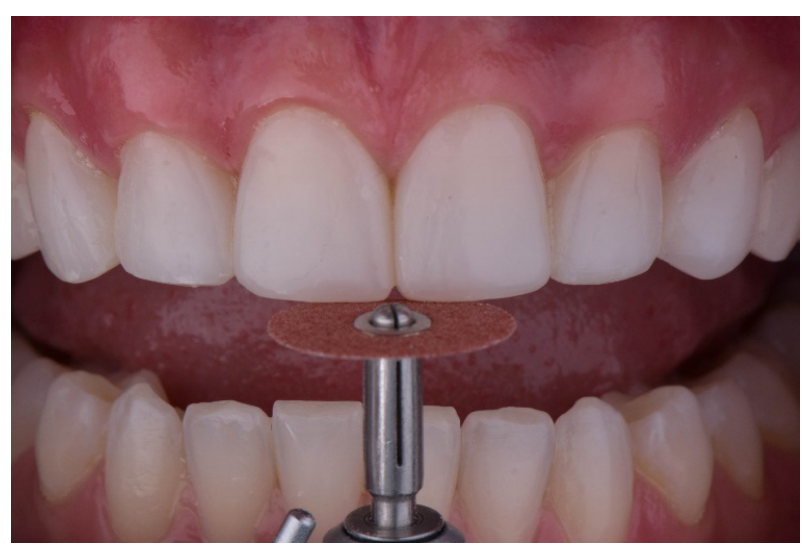

Figura 5 || Definição da curva do sorriso ascendente para distal.

Para melhor visualização das áreas planas vestibulares e convexas proximais, os terços cervical, médio e incisal da face vestibular das facetas, bem como as linhas de reflexão, foram demarcados com grafite colorido. Em seguida, um compasso de ponta seca foi utilizado para verificar o tamanho e simetria dos dentes homólogos (Figura 6). Este passo clínico 
é fundamental, uma vez que a distância entre as linhas de brilho determinam a percepção virtual do comprimento e largura dental. ${ }^{1,4}$ Nesse sentido, os terços dentais e as linhas de reflexão de luz foram reposicionados utilizando discos abrasivos de granulação grossa e média (Sof-Lex laranja escuro e laranja, 3M ESPE, São Paulo, Brasil) inclinados em $45^{\circ}$ (Figuras 7 e 8). Para obter maior compatibilidade com o formato dolicofacial da paciente, as facetas foram caracterizadas em formato oval suave.

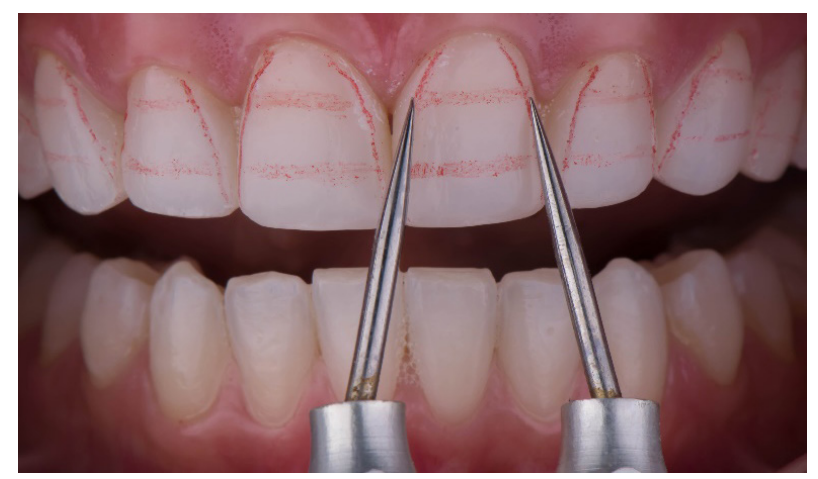

Figura 6 || Verificação da simetria dos dentes homólogos.

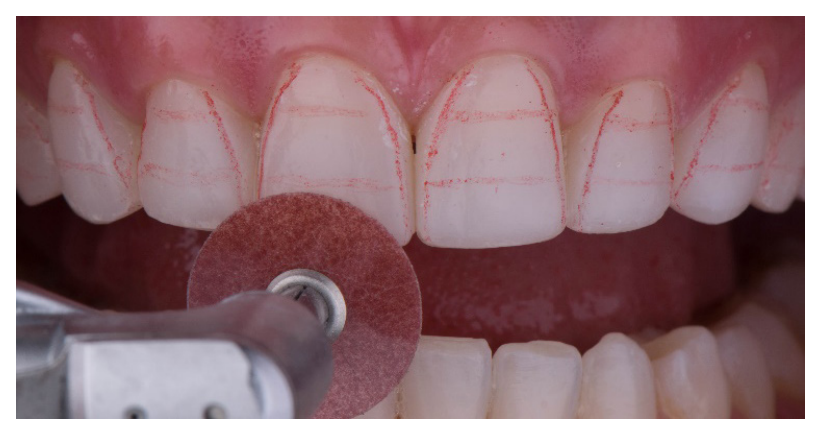

Figura 7 | Reposicionamento dos terços dentais.

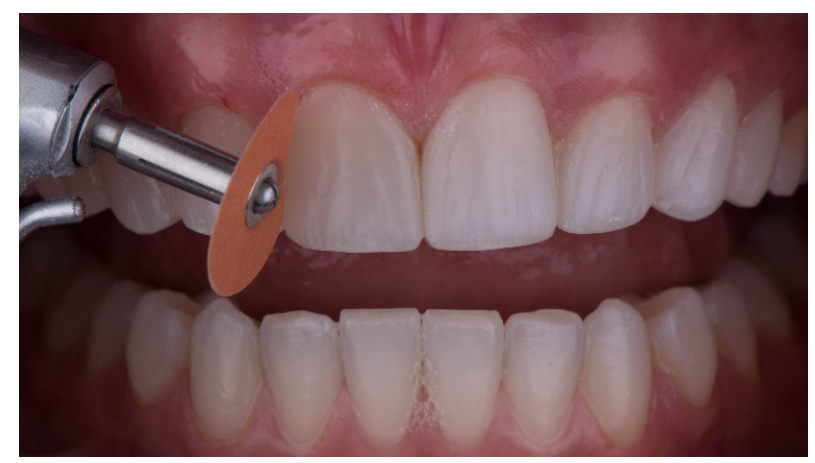

Figura 8 | Reposicionamento das linhas de reflexão de luz.
Finalizando a etapa de acabamento, as ameias incisais localizadas abaixo do ponto de contato foram redefinidas. Entre os incisivos centrais nota-se um formato triangular acentuado $\left(90^{\circ}\right)$ e a medida que se afastam da linha média, os ângulos incisais mesial e distal são arredondados, sendo o distal mais proeminente. Para a determinação das ameias incisais foram utilizados discos de granulação média e fina (Sof-Lex laranja e laranja claro, 3M ESPE, São Paulo, Brasil ) pois o desgaste não era acentuado.

\section{Texturização: anatomia secundária e terciária}

A determinação da anatomia secundária consiste, num primeiro momento, em confeccionar sulcos verticais e lóbulos de desenvolvimento da face vestibular. Essa microestrutura possui um formato triangular com a base voltada para incisal. Dos dois sulcos, o mesial é ligeiramente mais longo, estendendo-se ao terço médio dental. Na anatomia terciária, sulcos horizontais extremamente rasos são realizados principalmente no terço cervical, mimetizando as periquemáceas do esmalte. Para a texturização, foram utilizadas pontas diamantadas $\mathrm{F}$ e FF montadas em baixa rotação ( $n^{0}$ 2135 F e 1190FF - KG Sorensen, São Paulo, Brasil) (Figura 9). Em seguida, utilizando borrachas abrasivas impregnadas com óxido de alumínio (Enhance, Dentsply, Petrópolis, Brasil), os sulcos foram suavizados para não resultar em aspecto artificial.

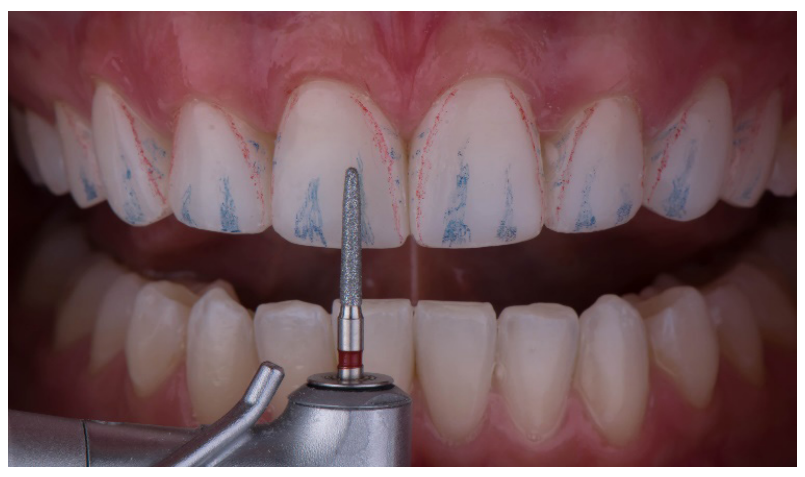

Figura 9 | Confecção dos sulcos de desenvolvimento. 


\section{Polimento}

A etapa final do protocolo consistiu em produzir facetas com superfícies brilhantes, semelhante ao esmalte natural. Para isso, borrachas abrasivas $\left(\mathrm{n}^{\text {os }}\right.$ 9150 e 9837, Jota, Suiça) e escova de carbeto de silício (Ivoclar Vivadent, Schaan, Liechtenstein) (Figura 10) foram utilizadas. A execução deve ser realizada em duas etapas, inicialmente, para que haja impregnação da pasta de polimento na superfície do material restaurador, deve-se utilizar alta pressão e velocidade baixa no motor contra-ângulo. Posteriormente, para obtenção do brilho final, o polimento é realizado com velocidade mais alta e baixa pressão em movimentos uniformes e intermitentes.

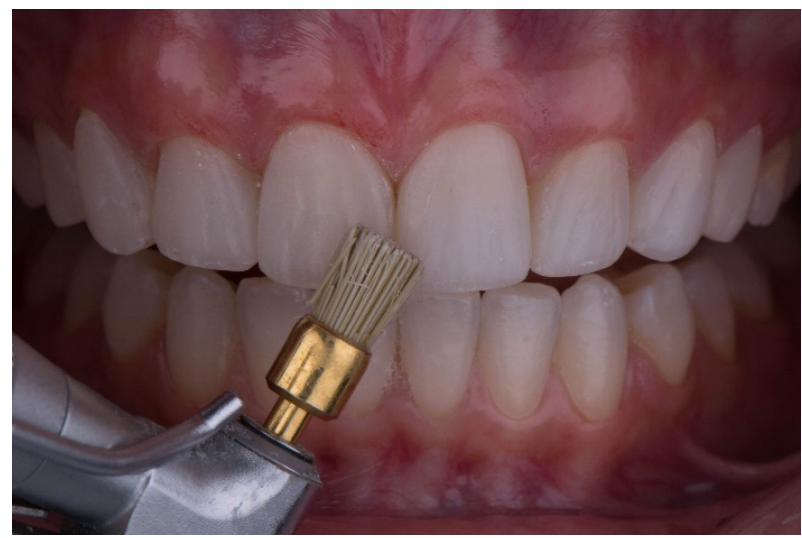

Figura 10 | Polimento das facetas diretas em resina composta.

O aspecto final estético e funcionalmente satisfatório das facetas diretas em resina composta após a execução do protocolo de acabamento, texturização e polimento é ilustrado na Figura 11.

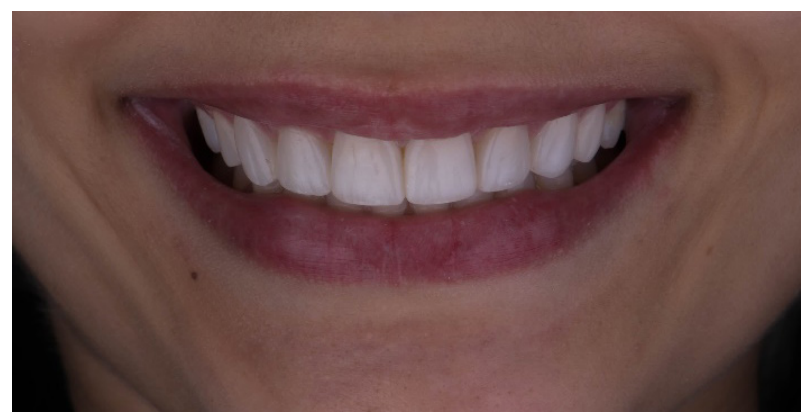

Figura 11 | Aspecto do sorriso após o protocolo de acabamento, texturização e polimento.

\section{DISCUSSÃO}

O presente caso clínico relata uma abordagem consciente e conservadora para otimização de facetas diretas em resina composta. No caso de restaurações com sobrecontorno, um protocolo previsível e reprodutível de acabamento e polimento foi suficiente para melhoria da anatomia e textura superficial das restaurações antigas. Isto é, um sorriso equilibrado foi conquistado sem a necessidade de substituir as restaurações, preservando a paciente de ser submetida à um tratamento desnecessário.

A condução do tratamento de restaurações antigas vem sendo retratada como desafiadora e a principal dúvida dos profissionais a respeito delas é quando repará-las, substituí-las ou acompanhálas. ${ }^{10}$ Nesse sentido, pode equivocadamente ocorrer a substituição de restaurações passíveis de reparo, fazendo com que o paciente entre em um ciclo restaurador repetitivo. ${ }^{2}$ A compreensão e identificação dos fatores de risco de cada paciente, bem como os motivos que determinaram a situação clínica, podem facilitar a tomada de decisão e postergar intervenções invasivas desnecessárias. ${ }^{2}$

Como todo material, restaurações em resina composta apresentam limitações e, embora a cárie adjacente às restaurações e fratura dental possam ocorrer, ${ }^{2,10}$ preocupações estéticas geralmente motivam reparos e/ou substituição de restaurações em dentes anteriores, ${ }^{4}$ uma vez que defeitos relacionados à alteração de cor são facilmente detectados pelos pacientes. ${ }^{2}$ Diante dessas circunstâncias, o acompanhamento clínico pós-operatório é extremamente importante para aumentar a taxa de sucesso das intervenções. Abordagens simples como o repolimento de restaurações podem contribuir para manutenção de uma superfície lisa e menos susceptível a retenção de biofilme e corantes, diminuindo o desenvolvimento de pigmentações extrínsecas. ${ }^{2,3}$

Dessa maneira, procedimentos de acabamento e polimento são fundamentais tanto para a 
concretização ideal do resultado estético e funcional esperado quanto e sua longevidade..$^{11,12}$ Diversas técnicas e materiais são indicados com esse propósito, porém, neste caso clínico o objetivo foi discorrer sobre uma sequência clínica criteriosa capaz de ser reproduzida pelo cirurgião-dentista que deseja otimizar os procedimentos restauradores estéticos.

Primeiramente, nesse protocolo, o clínico deve se preocupar em garantir a obtenção de facetas com adequado perfil de emergência $\left(45^{\circ}\right)$, bem adaptadas e com superfícies lisas que facilitem a higienização, garantindo menor acúmulo de biofilme, inflamação gengival e também alteração precoce de cor. ${ }^{11}$ Para obter restaurações menos rugosas, o sistema de acabamento utilizado deve possuir partículas abrasivas mais duras do que as partículas de carga presentes no material restaurador. ${ }^{12}$ Nesse sentido, evidências científicas mostram que discos contendo partículas com óxido de alumínio como Sof-Lex proporcionam melhores resultados do que os compostos por carboneto de silício, uma vez que conseguem desgastar as partículas de carga e a matriz orgânica de maneira similar. ${ }^{11,12}$

O próximo passo clínico consiste na visualização e posicionamento das linhas de reflexão. A verificação das distâncias entre as linhas de brilho mesial e distal é muito importante, pois elas definem o formato dental. ${ }^{1}$ Geralmente, o formato dos dentes segue o formato facial: oval, retangular ou triangular. Quando as linhas de reflexão convergem do terço médio para cervical e incisal, proporcionamos à restauração um formato ovóide; quando são paralelas entre si, retangular; e quando se apresentam divergentes tanto para cervical quanto para incisal, triangular. ${ }^{1}$ Por isso, cabe ao cirurgiãodentista identificar as características do paciente e reproduzi-las similarmente nos dentes restaurados.

Nesse cenário, a mimetização das caracterizações superficiais que um dente natural possui também é extremamente relevante. A confecção de sulcos verticais e horizontais na face vestibular da restauração proporciona uma superfície com textura, capaz de refletir melhor a luz incidente, contribuindo para a percepção estética do sorriso. ${ }^{1,12}$ Encerrando o protocolo, o polimento de toda superfície com borrachas abrasivas, escovas (carbeto de silício, pelo de cabra ou crina de cavalo) ou discos de feltro associados a pasta diamatanda possibilita brilho e lisura compatíveis com o esmalte dental, ${ }^{5,11}$ favorecendo a manutenção estética do tratamento restaurador.

\section{CONCLUSÃO}

A execução do protocolo de acabamento proposto, a texturização e o polimento podem ser uma boa alternativa para solucionar casos clínicos estéticos insatisfatórios. Essa abordagem foi capaz de melhorar a harmonia do sorriso, destacando características que favorecem a cor e o formato dental. A saúde periodontal e a estética foram otimizadas sem a necessidade de expor a paciente à tratamentos mais invasivos, como reparo ou substituição das facetas em resina composta.

\section{REFERÊNCIAS}

1. Sapata A, Sato C. Simple: uma abordagem simples em resinas compostas: anatomia, escultura e protocolos clínicos. Nova Odessa: Napoleão; 2017. 512 p.

2. Demarco FF, Collares K, Correa MB, Cenci MS, Moraes RR, Opdam NJ. Should my composite restorations last forever? Why are they failing? Braz Oral Res. 2017 Aug;31(1):e56. doi: 10.1590/1807-3107BOR-2017.vol31.0056.

3. Ardu S, Duc O, Di Bella E, Krekci I, Daher R. Correction to: Color stability of different composite resins after polishing. Odontology. 2018 Feb;16(3):328-33. doi: 10.1007/s10266018-0350-9.

4. Demarco FF, Collares K, Coelho-de-Souza FH, Correa MB, Cenci MS, Moraes RR, et al. Anterior composite restorations: a systematic review on long-term survival and reasons for failure. Dent Mater. 2015 Oct;31(10):1214-24. doi: 10.1016/j. dental.2015.07.005.

5. Erdemir U, Sancakli HS, Yildiz E. The effect of one-step and multi-step polishing systems on the surface roughness and microhardness of novel resin composites. Eur J Dent. 2012 Apr;6(2):198-205. 
6. Gönülol N, Yilmaz F. The effects of finishing and polishing techniques on surface roughness and color stability of nanocomposites. J Dent. 2012 Dec;40(2):e64-70. doi: 10.1016/j. jdent.2012.07.005.

7. Mondelli J, Furuse AY, Mondelli RFL, Mondelli AL. Estética e Cosmética em Clínica Integrada Restauradora. 2nd ed. São Paulo: Quintessence; 2018. 476p.

8. Croll BM. Emergence profiles in natural tooth contour. Part II: clinical considerations. J Prosthet Dent. 1990 Apr;63(4):374-379. DOI: 10.1016/0022-3913(90)90223-Y.

9. Tarnow DP, Magner AW, Fletcher P. The effect of the distance from the contact point to the crest of bone on the presence or absence of the interproximal dental papilla. J Periodontol. 1992 Dec;63(12):995-996. doi: 10.1902/jop.1992.63.12.995.
10. Wilson N, Lynch CD, Brunton PA, Hickel R, Meyer-Lueckel H, Gurgan S, et al. Criteria for the replacement of restorations: Academy of Operative Dentistry European Section. Oper Dent. 2016 Sep;41(S7):S48-57. doi: 10.2341/15-058-O.

11. Aytac F, Karaarklan ES, Agaccioglu M, Tastan E, Buldur M, Kuyucu E. Effects of novel finishing and polishing systems on surface roughness and morphology of nanocomposite. J Esthet Restor Dent. 2016 Jul;28(4):247-261. doi: 10.1111/ jerd.12215.

12. Erdemir U, Esra Y, Meltem ME, Alev O, Fulya TT. Effects of polishing systems on the surface roughness of tooth-colored materials. J Dent Sci 2013 Jun;8(2): 160-169. doi: 10.1016/j. jds.2012.05.007. 